\title{
Perceções dos estudantes do ensino superior sobre o papel da responsabilidade social organizacional: Um estudo exploratório
}

\section{Perceptions of higher education students about the role of corporate social responsibility: An exploratory study}

\author{
Joana Lobo Fernandes*, Cláudia Andrade** \\ *Escola Superior de Educação, Politécnico de Coimbra, ** Escola Superior de Educação, Politécnico de Coimbra
}

\begin{abstract}
Resumo
$\mathrm{O}$ presente estudo analisou, numa amostra de 70 estudantes da licenciatura em Comunicação Organizacional, as perceções sobre o papel da responsabilidade social organizacional e o modo como as organizações podem atuar, junto dos colaboradores, recorrendo às novas tecnologias de informação e comunicação. A análise correlacional entre as dimensões das escalas escala de perceção do papel de responsabilidade social (RS) e da escala de expetativas de comunicação fora de horário de trabalho permitiu identificar uma correlação positiva e significativa entre a RS e sucesso e competitividade (obstáculo) e as expetativas de comunicação para além do horário de trabalho. Os resultados são discutidos no âmbito da formação académica promotora de reflexão e ação orientadas para a valorização da sustentabilidade organizacional e o bem-estar do colaborador.

Palavras chave: estudantes, ensino superior, formação
\end{abstract}

\begin{abstract}
The present study analyzed, in a sample of 70 undergraduate students og Organizational Communication, their perceptions about the role of organizational social responsibility and how organizations can interact with employees using new information and communication technologies. The correlational analysis between the dimensions of the scales of perception of the role of social responsibility (SR) and the scale of expectations of communication outside working hours allowed found a positive and significant correlation between SR and success and competitiveness (obstacle) and communication expectations during off-work hours. The results are discussed within the framework of the academic formation that promotes reflection and action oriented towards the valorization of organizational sustainability and the well-being of the employee. Keywords: Students, higher education, formation
\end{abstract}

\section{Introdução}

As mudanças que ocorrem nos contextos organizacionais sugerem a atualização do modo de preparar os futuros profissionais para que estes respondam adequadamente aos novos desafios. As alterações decorrentes dos desenvolvimentos tecnológicos colocam questões pertinentes aos modos de trabalhar em contexto profissional, e de agir em contexto organizacional que interessa analisar. A responsabilidade social organizacional, enquanto temática de reflexão fundamental e transversal ao domínio dos estudos organizacionais, oferece um quadro de leitura pertinente para analisar as perceções dos estudantes (futuros profissionais) em relação aos impactos das novas tecnologias na gestão do tempo e do espaço de trabalho. Nos últimos anos, a investigação sobre a responsabilidade social organizacional deixou de se centrar apenas nos contextos empresariais para passar a prestar especial atenção ao modo como os contextos de formação, nomeadamente ao nível do ensino superior, podem promover de forma ativa o desenvolvimento de competências e sentido de agência neste domínio. Neste estudo, de cariz exploratório e quantitativo, procuramos compreende as perceções dos estudantes sobre o papel da responsabilidade social organizacional, destacando, ao nível da dimensão interna, a avaliação do modo como as organizações podem atuar, junto dos colaboradores, recorrendo às novas tecnologias de comunicação e informação.

Responsabilidade social das organizações e contextos formativos

A responsabilidade social das organizações apresenta-se como um tema incontornável para o mundo dos negócios (Crowther \& Aras, 2008), mas igualmente como ponto de partida para uma reflexão sobre as práticas que ocorrem nesse contexto, colocando-se como paradigma alternativo aos modelos tradicionais da gestão (Laszlo, Sroufe \& Waddock, 2017). Embora não exista uma definição única, na Europa o referencial predominante sublinha que a RSO (ou RSE) corresponde à integração voluntária de preocupações sociais e ambientais e uma orientação para as expetativas dos diferentes stakeholders, sublinhando que o conceito se desdobra nas dimensões interna e externa (CCE, 2001) ou ainda que a RSO representa a tomada de consciência dos diferentes impactos gerados e a assunção das responsabilidades daí decorrentes (CE, 2011). Nesta lógica, a RSO não é um mero acrescento às práticas 
tradicionais de gestão mas um novo paradigma para (re)pensar as práticas gestionárias (Neves, 2004).

No presente artigo, importa destacar a dimensão interna da RSO, porquanto o mesmo incide sobre as perceções que os inquiridos (aqui, atuais estudantes de comunicação organizacional) têm relativamente ao impacto das novas tecnologias em contexto laboral no momento em que perspetivam a sua entrada no mercado de trabalho. Assim, a temática incide na relação laboral, enquanto espaço de exercício de direitos e deveres e de articulação entre as esferas pessoal/familiar e laboral. Enquanto stakeholder, o público interno protagoniza um conjunto de aspetos, na relação com a entidade empregadora que vão desde os processos de recrutamento aos de aposentação, passando pelas questões de equidade salarial e de oportunidade de carreira, conciliação trabalho/família, formação contínua ou ainda a preparação para a demissão (Instituto Ethos, 2017). Destaque particular para o tema "Condições de trabalho, Qualidade de vida e Jornada de trabalho" (Instituto Ethos, 2017, p. 56) que engloba o "respeito e limites com relação ao trabalho remoto, como por exemplo, telefonemas e e-mails fora do horário de trabalho", preconizando que a organização deva ser reconhecida quando implementa boas práticas na definição de uma jornada de trabalho equilibrada" (Instituto Ethos, 2017, p. 56).

Por outro lado, e entendendo que um dos eixos de atuação (e de preocupação) das instituições de ensino superior é o de oferecer uma formação profissional e cidadã (Vallaeys et al., 2009), a vertente de cidadania traduz-se na "presença de temáticas cidadãs e de responsabilidade social no currículo (diretos humanos, desenvolvimento sustentável, ética profissional e cívica, gestão da RS, entre outros" (Vallaeys et al., 2009, p. 33), procurando responder ao desafio de formar cidadãos responsáveis que fomentem um desenvolvimento mais humano e sustentável (Vallaeys et al., 2009, p. 32). Assim se compreende que uma abordagem à RSO em contexto de formação académica se constitui como uma oportunidade para que o aluno perspetive a sua atuação futura, em contexto laboral e na sociedade em geral, interiorizando princípios alinhados com os principais referenciais que norteiam as organizações (sobretudo as empresas) socialmente responsáveis, nas matérias dos direitos humanos (com o referencial da DUDH $^{1}$ ), das práticas de um trabalho decente (convenções da $\mathrm{OIT}^{2}$ ) e integrando o desenvolvimento da pessoa na sustentabilidade ambiental (Declaração do $\mathrm{Rio}^{3,4}$ ). Na mesma linha, o movimento da RME (Responsible Management Education) nas Escolas de Negócios questiona a formação ministrada colocando a tónica na importância de implementar mudanças futuras ${ }^{5}$ e evitar novas crises dos mercados e da sociedade (Storey et. al,

\footnotetext{
${ }^{1}$ Declaração Universal dos Direitos Humanos (1948)

${ }^{2}$ Organização Internacional do Trabalho (1998)

${ }^{3}$ Aprovada na Cimeira do Rio (1992)

${ }^{4}$ Importa indicar ainda o Pacto Global das Nações Unidas que resulta da fusão destes três documentos referenciais e ainda da Convenção das Nações Unidas contra a Corrupção.

${ }^{5}$ É preconizado um alinhamento com a realização dos Objetivos de Desenvolvimento Sustentável (2016-2030) da ONU (Storey et al. 2017)
}

2017), o que passa por uma revisão do raciocínio dominante que era privilegiado, e ministrado, nessas instituições de ensino (de uma "narrativa neoliberal" para uma outra, de uma "economia emergente ao serviço da vida", segundo Laszlo et al., 2017, pp. 108-110).

Por conseguinte, uma análise das perspetivas que os estudantes de comunicação organizacional apresentam no âmbito da atuação ao nível da responsabilidade social organizacional pode permitir uma mais valia de modo a promover uma formação mais completa que possibilite a atuação em consonância destes futuros profissionais. Atinge-se assim um objetivo duplo: de sensibilização dos estudantes para a importância da RSO e de tomada de consciência dos comportamentos que devem ser esperados e exigidos, em contextos laborais onde uma atuação socialmente responsável é cada vez mais um requisito.

Responsabilidade Social Organizacional - dimensão interna: impactos uso das tecnologias de informação e comunicação

O recurso a meios tecnológicos de informação e comunicação cada vez mais diversificados por parte das organizações gerou alterações nas relações entre estas e os seus colaboradores (Daft, 2006; Turel, Serenko \& Bontis, 2011). Como referem Piszczek, Pichler, Turel, e Greenhaus (2016) as tecnologias de informação e comunicação como os smartphones, tablets e os computadores portáteis estão presentes no quotidiano no contexto das sociedades industrializadas, fazendo, por vezes, esbater a outrora visível fonteira entre o trabalho e a vida pessoal do colaborador. Reconhecidas as potencialidades e benefícios decorrentes do uso das tecnologias de informação e comunicação nos contextos organizacionais, o seu uso mais frequente, em diferentes espaços, veio desencadear um conjunto de estudos que destacam os potenciais efeitos nefastos, para os colaboradores, do uso excessivo ou inadequado das tecnologias de informação e comunicação. Por exemplo, o estudo de Day, Paquet, Scott, e Hambley (2012), refere que o uso das tecnologias de informação e comunicação no espaço privado (por exemplo, em casa) ou noutros contextos sociais pode trazer efeitos negativos para o colaborador uma vez que aumenta a probabilidade de estes sere manterem ativos relativamente à necessidade de responder às exigências profissionais. $\mathrm{Na}$ atualidade, $\mathrm{e}$ com a evolução para smartphones esta realidade não só continua a fazer-se sentir como pode ainda ser ampliada, uma vez que os smartphones apresentam ainda mais potencialidades de conectividade. Currie e Eveline (2011) analisaram o uso desta tecnologia (smartphones) entre profissionais académicos. Os resultados evidenciaram que o e-mail era consultado, por estes, de forma imediata, após a sua receção através do smartphone. Este estudo observou, ainda, que a vida pessoal pode ser influenciada negativamente devido ao uso das tecnologias, podendo mesmo causar situações de stresse (Currie \& Eveline, 2011). Uma das consequências desta forma mais flexível de trabalhar que as tecnologias nos oferecem é a cultura do "sempre disponível", ou seja, instituir-se, de algum modo, a perceção de que o colaborador pode estar sempre disponível para trabalhar 
mesmo não estando no seu horário laboral (Currie \& Eveline, 2011). Ao serem utilizadas de forma abusiva as tecnologias de informação e comunicação acabam por fazer com que os colaboradores estejam sempre ligados com os seus smartphones acabando por trabalhar mais horas chegando, em alguns casos a tornar a semana de trabalho mais longa (Thurston, 2012). Consequentemente, têm menos tempo para dedicar a vida pessoal com efeitos nefastos ao nível do bem-estar podendo gerar, por exemplo, exaustão emocional e conflito de papéis (Derks \& Bakker., 2014; Piszczek et al.,2016; Thurston, 2012).

\section{Método}

\section{Participantes}

Os dados deste estudo foram recolhidos junto de uma amostra de 70 estudantes do ensino superior a frequentar a licenciatura em Comunicação Organizacional, Escola Superior de Educação, em regime diurno e pós-laboral e de ambos os sexos (18.8\% homens e $76.8 \%$ mulheres). As idades variam entre um mínimo de 18 anos e um máximo de 49 , sendo a média de idades de 23.88 anos ( $\mathrm{DP}=7.53$ ). No que se refere ao ano de frequência da licenciatura a maioria frequenta o $2^{\circ}$ ano do curso (83\%). Dos restantes, $8.7 \%$ frequentam o $1^{\circ}$ ano e $8.3 \%$ o $3^{\circ}$ ano da licenciatura. Constata-se, ainda, que $43.5 \%$ afirmam ser apenas estudantes e $56.5 \%$ são trabalhadores-estudantes ou estudantes que trabalham ocasionalmente (p. exemplo, ao fim de semana, alguns dias por semana, nas férias escolares, etc).

\section{Instrumentos}

Perceções acerca do papel da responsabilidade social. Baseada na escala originalmente desenvolvida por Singhapadki, Kraft, Vitell e Rallapalli (1995), Elias (2004) apresenta uma versão adaptada da escala constituída por 13 itens organizada em 2 fatores: 4 itens para a dimensão do lucro, 6 itens para a dimensão do sucesso a longo prazo e 3 itens para a dimensão do sucesso a curto prazo. Os itens foram respondidos numa escala de Likert com cinco pontos $(1=$ discordo totalmente até $5=$ concordo totalmente). A análise fatorial exploratória em componentes principais, após rotação varimax, permitiu encontrar uma solução em dois fatores (tendo sido eliminado o item 12 da escala original, devido a baixa saturação, inferior a 0.50 , em ambos os fatores), diferindo assim, da organização em quatro fatores da escala original. As contribuições dos dois fatores para a variância explicada são, respetivamente, $26 \%$ e $15 \%$. A medida de Kaiser-Meyer-Olkin (KMO), obteve um coeficiente de 0.77, e o Teste de Esfericidade de Bartlett indicou um valor de 1103.16 evidenciando a adequação dos dados em análise são adequados para realização da análise fatorial (Marôco, 2007). A nova reorganização dos fatores, resultante das saturações fatoriais, integra itens no fator 1 referente à responsabilidade social e competitividade (obstáculo) e responsabilidade social e sucesso no fator 2 , estando os dois fatores correlacionados negativamente $(\mathrm{r}=.35)$. A análise da consistência interna para escala global apresenta valor de $\alpha=.87$ e de $\alpha=.81$ para o fator 1 (responsabilidade social e competitividade) e de $\alpha=.81$ para o fator 2 (responsabilidade social e sucesso).

Expetativas de comunicação com a organização fora de horário de trabalho. No presente estudo adaptou-se a escala usada Piszczek (2014) que avalia as expetativas de comunicação com a organização para além do horário de trabalho. Os sete itens da escala original, depois de traduzidos para português foram respondidos numa escala de Likert com cinco pontos ( $1=$ discordo totalmente até $5=$ concordo totalmente). A escala revelou-se, através da análise fatorial exploratória, unidimensional, tal como a escala original. O cálculo do coeficiente alfa de Cronbach, para análise da consistência interna, indicou a presença de um valor muito bom $(\alpha=.92)$.

\section{Procedimento}

O método de recolha de dados escolhido foi um questionário on-line com recurso à plataforma Google Forms, difundido por email institucional aos estudantes da licenciatura em Comunicação Organizacional da Escola Superior de Educação de Coimbra, sendo solicitada a participação no estudo.

\section{Resultados}

Antes de se proceder ao estudo correlacional, foi efetuado, com recurso ao programa Statistical Package for Social Siences (v. 21, SPSS Inc., Chicago, IL), o estudo da distribuição das variáveis, utilizando quatro critérios: Coeficientes de assimetria e curtose, Teste de ajustamento de Kolmogorov-Smirnov. Verificou-se que as variáveis em estudo seguem uma distribuição aproximadamente normal. Deste modo, numa primeira etapa foi efetuada uma análise descritiva que permitiu obter os valores médios e o desvio-padrão das variáveis em estudo, assim como as correlações de Pearson, e o respetivo teste de significância, entre as variáveis. A média e o desvio-padrão indicam, relativamente à responsabilidade social e competitividade (obstáculo) $(\mathrm{M}=4,24 ; \mathrm{DP}=.59)$ que os participantes escolheram em média a resposta "Concordo". No que diz respeito à responsabilidade social e sucesso $(\mathrm{M}=4,31 ; \mathrm{DP}=.87)$, a resposta média foi "Concordo". Já em relação à expetativas de comunicação com a organização fora de horário de trabalho $(\mathrm{M}=4,35 ; \mathrm{DP}=.61)$ a resposta média que os participantes deram foi também "Concordo". As correlações entre as duas dimensões da escala de perceções acerca do papel da responsabilidade social e as expectativas de comunicação com a organização fora do horário de trabalho revelaram a existência de uma correlação significativa e positiva entre responsabilidade social e sucesso e competitividade (obstáculo) e as expetativas de comunicação para além do horário de trabalho $(\mathrm{r}=.64, \mathrm{p}<.001)$.

\section{Discussão e Conclusões}

No que diz respeito aos resultados da estatística descritiva podemos verificar que os estudantes que participaram no presente estudo valorizam ambas as 
dimensões da responsabilidade social: responsabilidade social e competitividade (obstáculo) e responsabilidade social e sucesso, o que reflete, de algum modo a coabitação de visões não consensuais uma vez que são valorizadas pelos mesmos estudantes tanto perspetivas de apologia da responsabilidade social organizacional como visões que a colocam enquanto entrave ao sucesso organizacional. De igual modo os estudantes apresentam valores no campo da concordância ao nível das expetativas de comunicação, através dos meios eletrónicos para além do horário de trabalho, sublinhando uma postura de aceitação desta realidade. Para além destes aspetos também foram encontradas algumas relações positivas e significativas entre as dimensões estudadas, que foram evidenciadas pelas correlações de Pearson. Verifica-se que para os estudantes de comunicação organizacional existe uma correlação significativa e positiva entre responsabilidade social e competitividade (obstáculo) e as expetativas de comunicação para além do horário de trabalho. Tal facto remete-nos para a necessidade de reflectir, novamente, sobre a aparente aceitação, por parte dos estudantes, de práticas organizacionais que apontam para uma jornada de trabalho que extravasa o horário laboral para ocupar um espaço privado, familiar, de lazer e de descanso, sem uma (aparente) contestação, assumindo como uma consequência decorrente da omnipresença dos dispositivos eletrónicos na vida do sujeito. $\mathrm{O}$ facto de um mesmo instrumento - por exemplo, o smartphone - ser entendido como uma extensão natural do ser humano, quer nos contextos de trabalho quer nas relações interpessoais, parece gerar um comportamento de aceitação sem questionamento de práticas laborais fora dos locais e horários de trabalho.

Dado que se trata de um estudo efetuado junto de futuros profissionais, destaca-se que no âmbito da formação no ensino superior o desenvolvimento de competências em domínios específicos relacionados com as práticas organizacionais reveste-se de um caráter académico que muitas vezes só permite a sua aplicação no âmbito dos estágios ou de práticas em projetos de intervenção no âmbito da própria instituição. $\mathrm{O}$ desenvolvimento de práticas de estudos de caso e/ou simulação poderão ser estratégias a desenvolver de modo a capacitar os estudantes de sentido crítico e de capacidade de agência. O desenvolvimento destas capacidades pode ser feito através do desenho de uma articulação curricular que fomente abordagens integradoras de saberes para os quais concorrem, de forma ativa, a formação em responsabilidade social organizacional, em ética empresarial mas igualmente na aquisição de uma visão integradora sobre as problemáticas sociais globais. A formação para um pensamento crítico, uma cidadania ativa e uma aprendizagem baseada na participação em projetos sociais, parece ser uma forma para se consolidarem competências de respeito pelo outro e de valorização da diversidade.

A natureza exploratória deste estudo, deve ser sublinhada, pelo que os resultados encontrados são apenas indicadores para que seja realizado, posteriormente, um estudo mais alargado dos instrumentos utilizados com um maior número de participantes.

\section{Referencias}

CCE - Comissão das Comunidades Europeias (2001). Livro Verde Promover um quadro europeu para a responsabilidade social das empresas. Bruxelas: CCE. CE - Comissão Europeia (2011). Corporate Social Responsibility: a new definition, a new agenda for action. Memo 11/730/2011 de 25 de Outubro. Bruxelas: CE.

Crowther, D. \& Arras, G. (2008). Corporate social Responsibility (PDF). Retirado de www. bookboon.com.

Currie, J., \& Eveline, J. (2011). E-technology and work/life balance for academics with young children. Higher Education: The International Journal of Higher Education and Educational Planning, 62(4), 533-550. doi: 10.1007/s10734-010-9404-9

Daft, R. L. (2006). The new era of management. USA: Thompson

Day, A., Paquet, S., Scott, N., \& Hambley, L. (2012). Perceived information and communication technology (ICT) demands on employee outcomes: The moderating effect of organizational ICT support. Journal of Occupational Health Psychology, 17(4), 473-491. doi: 10.1037/a002983

Derks, D., \& Bakker, A. B. (2014). Smartphone use, work-home interference, and burnout: A diary study on the role of recovery. Applied Psychology: An International Review, 63, 411-440. doi: 10.1111/j.1464-0597.2012.00530.x

Instituto Ethos de Empresas e Responsabilidade Social (2017). Indicadores Ethos para Negócios Sustentáveis e Responsáveis (ciclo 2016/17). São Paulo: Instituto Ethos.

Laszlo C., Sroufe, R., \& Waddock, S. (2017). Torn between two paradigms: a struggle for the soul of Business Schools. AI Practitioner, 19 (2), 108-119. doi: 10.12781/978-1-907549-31-1-12

Piszczek,M., Pichler, S., Turel, O., \& Greenhaus, J. (2016). The information and communication technology user role: Implications for the work role and inter-role spillover. Frontiers in Psychology, 7, 1-15. doi: 10.3389/fpsyg.2016.02009

Storey, M., Killian, S., \& O'Regan, P. (2017).Responsible Management Education: Mapping the field in the context of SDGs. The International Journal of Management Education, 15, 93-103. doi: 10.1016/j.ijme.2017.02.009

Thurston, R. (2012). The technology threat to work/life balance. GPsolo, 29(5), 36-40.

Turel, O., Serenko. A., \& Bontis, N. (2011).Family and work-related consequences of addiction to organizational pervasive technologies. Information and Management, 48, 88-95. doi: 10.1016/j.im.2011.01.004

Vallaeys F., de la Cruz C., \& Sasia, P. (2009). Responsabilidad Social Universitaria: Manual de primeiros pasos. Mexico: McGraw-Hill Interamericana. 